\title{
Economic cascades and the costs of a business-as-usual approach to COVID-19
}

\author{
Peter D. Roopnarine*1, David Goodwin ${ }^{2}$, Maricela Abarca ${ }^{1}$, and Joseph \\ Russack $^{1}$ \\ ${ }^{1}$ Institute for Biodiversity Sciences and Sustainability, California Academy \\ of Sciences, 55 Music Concourse Dr., San Francisco CA 94118 \\ ${ }^{2}$ Department of Geosciences, Denison University, Granville OH 43023, USA
}

\begin{abstract}
Shelter-in-place policies and the closure of non-essential workplaces intended to disrupt transmission of the SARS-COV-2 virus are effective approaches to combating COVID-19. They have, however, caused record levels of unemployment in the United States, raising questions of whether mitigation is more societally damaging than the disease. Here we use a coupled epidemiological-economic model to estimate the impact on employment of an unmitigated, business-as-usual approach to the pandemic. We compared unemployment between March-August 2020 in ten Californian socioeconomic systems (SESs) to unemployment forecast by a model of industrial sector inter-dependencies subjected to unmitigated outbreaks of COVID-19. We found that economic losses are unavoidable because disease-driven losses propagate economically through SESs, amplifying losses to the disease. While model forecasts are generally lower than actual unemployment, jobs savings would come at the cost of greatly increased worker mortality. The costs would also be disproportionately greater among smaller and inland SESs.
\end{abstract}

Key words: COVID-19 | economic model | economic cascade | economic impact coronavirus | California economy

Plain language summary:We use a coupled epidemiological-economic model to predict the unemployment that would be incurred by major Californian socio-economic systems if outbreaks of COVID-19 were permitted to run their courses. This is a baseline against which it is important to compare contrasting approaches that prioritize either non-pharmaceutical actions intended to disrupt spread of the disease, or safeguards to uninterrupted economic activity. We find that high unemployment would be unavoidable as the effects of worker death or debilitating illness cascade

*proopnarine@calacademy.org 
through the economic network. While predicted unemployment is lower than actual unemployment during the pandemic, that benefit comes at the cost of greatly elevated mortality. The impact would also be disproportionately more severe among smaller, goods producing, and typically inland socioeconomic systems.

The current lack of effective or widely available treatments or vaccines for COVID-19 has prompted a variety of non-pharmaceutical interventions, intended to disrupt transmission and persistence of the disease. Efforts have focused on reducing the frequency of contact between infected and susceptible individuals, and lowering the probability of infection during episodes of contact. Contact reduction has been implemented in multiple ways, for example ranging from limits on the number of occupants in a workplace, to the complete shutdown of workplaces. Collectively those actions, whether coercive or dependent on societal compliance, have had negative economic impacts. The consequences have been severe in most cases, including globally high levels of unemployment, reductions of productivity, disruptions of trade and the movement of goods, and the possibility of a global recession (19). The scale of the economic downturn has exceeded downturns associated with previous pandemics (6), and forecasts of the impacts of future pandemics (13). The persistence of the disease in some countries, and its resurgence in others, threatens to extend economic recessions beyond 2020 (5) and has raised questions of whether efforts to manage the health emergency are justified given the consequential economic impacts. There are multiple viewpoints of this argument, e.g. predictions that social shutdowns during an influenza pandemic would be more economically damaging than a pandemic itself (13), in contrast to the conclusion that social distancing laws affect consumer spending to a lesser degree than the effect of COVID19 itself (25). An essential element in understanding the merits of any view or strategy is the extent to which the health emergency alone would affect an economy; that is, the economic impact of the disease itself in the absence of any economically impactful mitigation decisions. This is a baseline against which the economic benefits or costs of disease-mitigating efforts should be assessed, and would be the economic consequence of allowing an outbreak of COVID-19 to "run its course" through an economic system. We term this strategy a "business-as-usual" model.

Here we present a numeric business-as-usual model of the mutualistic relationships among major industrial sectors within the United States, employment within those sectors, and the expected rates of mortality and morbidity in the employed labor force given an outbreak of COVID-19. We applied the model to ten major metropolitan areas, or socio-economic systems (SESs) in the United States state of California, modeling outbreaks for a range of transmission intensities, including those estimated to have occurred within the areas. The model predicts the loss of employment within an SES driven by mortality and severe illness caused by COVID-19, as well as losses caused by the subsequent reductions of productivity and demand within the SES. Finally, we compared model forecasts based on the initial contagiousness of an outbreak $\left(R_{0}\right)$ to those that resulted from real mitigation efforts and reduced rates of transmission ("flattening the curve"; $R$-effective, or $R_{\text {eff }}$ ).

California SESs are metropolitan divisions (MDs) and statistical areas (MSAs) delineated by the United States Office of Management and Budget and utilized by the United States Bureau of Labor Statistics (USBLS) and the California Employment and Development 


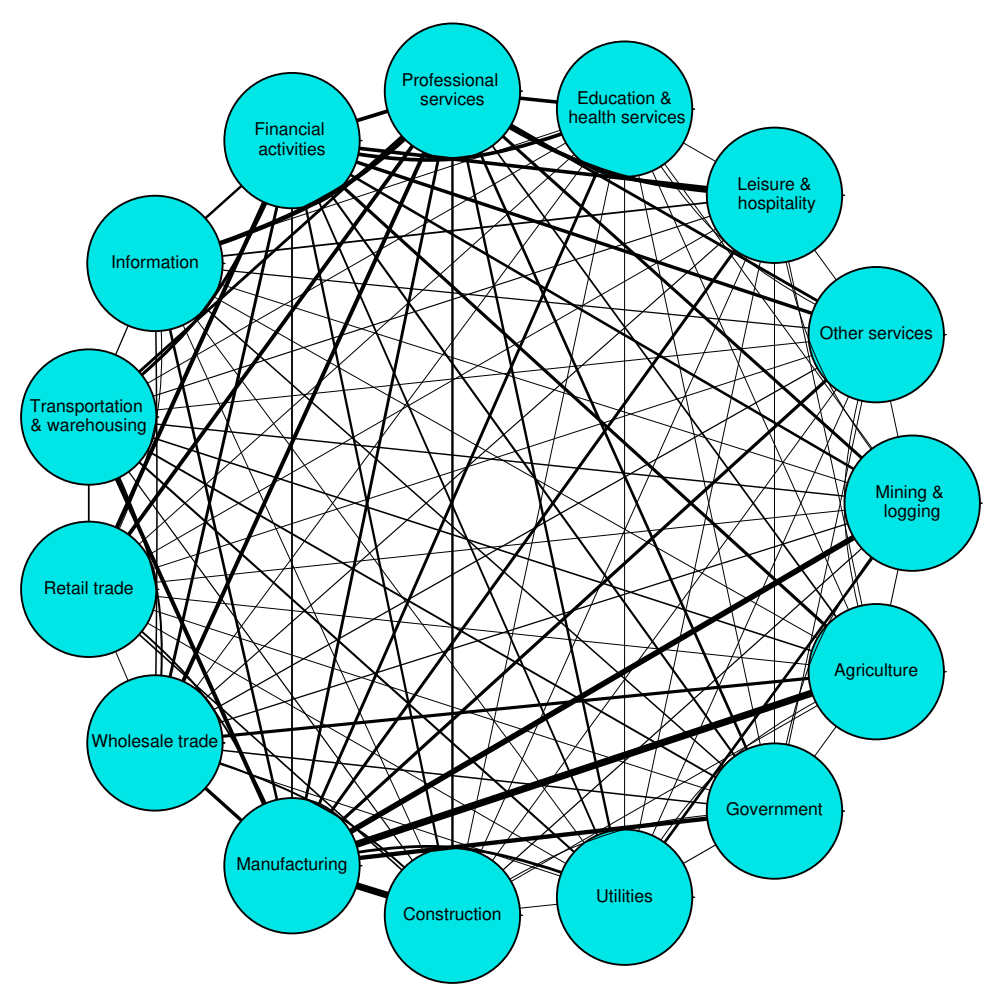

Figure 1: Socio-economic network of major industrial sectors. Link widths are scaled according to total inter-sector exchange strengths ( $\mu$ in Eq. 4; see Materials and Methods; Dataset $\mathrm{S} 2$ ). Thicker links indicate stronger inter-sector dependencies. Note that "Agriculture" here is equal to employment listed as "Total Farm" by the CAEDD. 
Department (CAEDD) for reporting of economic data. Our SESs include: San FranciscoSan Mateo-Redwood City MD, Oakland-Berkeley-Livermore MD, San Jose-Sunnyvale-Santa Clara MSA, Stockton-Lodi MSA, Fresno MSA, Los Angeles-Long Beach-Glendale MD, AnaheimSanta Ana-Irvine MD, Riverside-San Bernardino-Ontario MSA, Oxnard-Thousand OaksVentura MSA, and San Diego-Carlsbad MSA (20, 2) (Fig. S1). California presents a suitable case for examination because the state's economy is comparable in size to those of many nation states', ranking as the world's $5^{\text {th }}$ largest in 2019 (4). Yet, SESs within the state, including those used here, span an order of magnitude in size. The smallest SES examined, Stockton-Lodi, had a workforce of 258,300 employees in February, 2020, whereas the Los Angeles-Long Beach-Glendale SES's was 4,636,800. The SESs are also structurally diverse, comprising systems dominated alternatively by goods producing or services providing industries. Furthermore, California acted early during the emergence of the pandemic in the United States, declaring a state of emergency on March $4^{\text {th }}$, and statewide shelter-in-place orders, social distancing policies, and the closure of non-essential businesses on March $19^{\text {th }}$. The economic reaction was severe and nearly immediate, with more than 2 million workers in our SES set being unemployed by April. California's economy and its regional components may thus serve as suitable analogs for SESs elsewhere.

The business-as-usual CASES (Complex Adaptive Socio-Economic Systems) model developed here is a compartmental and network model that treats employment within an industrial sector as a function of employment levels in all sectors, as mediated by the value of inter-industry exchanges (Fig. 1). Changes of employment in any sector therefore have cascading consequences throughout the economic network. The business-as-usual scenario treats economic drivers of hiring and layoffs as constant, with the system being perturbed only by COVID-19-driven losses of workers because of severe illness or mortality. We simulated the scenario for each SES for 151 days, representing the interval from March to August, 2020, an interval for which consistent employment data are now available. Simulations were conducted under a broad range of potential outbreak intensities, accounting for the differential age-dependent impacts of COVID-19. Scenarios were also simulated for 365 days under the outbreak conditions that were particular to each SES on March 1st.

\section{Results and Discussion}

The model was simulated for each SES with a start date of March 1, 2020, when outbreaks were becoming widespread in California. Initial employment levels were therefore those recorded for the month of February, 2020. Age structure per SES sector were based on United States Census Bureau (USCB) estimates for the final quarter of 2019 (Dataset S1). Model outbreaks were initiated by infecting $0.002 \%$ of the general population, and then simulating a coupled SIR-CASES epidemiological-economic model (SIR - Susceptible, Infected, Removed) for 151 days (see Materials and Methods). Employment in each sector, and thus an entire SES, declined as workers were removed because of illness (Fig. 2A). There is little differentiation among sectors when cascades are initiated because the network is complete (that is, all nodes are connected) and changes in any sector propagate quickly throughout the network (Fig. S2). The magnitude of the decline of a sector within an SES, or job 
loss $\Delta E_{i}$ within sector $i$, depends on $R_{0}$ and the age distributions within sectors, and in all instances increased nonlinearly with increasing $R_{0}$. Sectors with greater proportions of older workers experienced greater and more rapid losses because of the disease's greater severity in older individuals (Fig. 2B). Those losses depend on SIR model forecasts, tracking the Removed compartment of the population, and the fraction of the general population that was employed in February, 2020 (see Materials and Methods). $\Delta E_{i}$ varied notably among SESs, however, driving the ways in which different systems responded to outbreaks, and the differences of total job losses among the systems (Fig. S2). We return to this point below.

The divergence, or gap, between the disease-only and economically propagated forecasts (Fig. 2C), as well as the magnitude of the cascade, are functions of both $R_{0}$ and SES network structure, and all the SESs examined exhibited the same type of response to outbreak (Fig 2D Fig. S3). The dependence of $\Delta E$ on $R_{0}$, and the resulting magnitude of $\Delta E$, however, vary among the SESs (Fig. $2 \mathrm{E}, \mathrm{F}$ ). Because $\Delta E$ asymptotes rapidly with increasing $R_{0}$, we summarized the expected reduction of employment in each SES as the growth rate and asymptotic value of $\Delta E$ on day 151 as a function of $R_{0}$ (Fig. S4). Both values were estimated from nonlinearly fitted logistic functions of the $\Delta E^{*}$ vs. $R_{0}$ profile on day 151 (Fig. S5; Table S1), where $\Delta E^{*}$ is final employment relative to initial employment $(\mathrm{E}(0))$. Forecasts of $\Delta E^{*}$ ranged from $95.6 \%$ of February, 2020 employment (RiversideSan Bernardino-Ontario) to $86.3 \%$ (Fresno). $\Delta E^{*}$ scales negatively with initial employment (Fig. 2E,F), with larger systems experiencing relatively less unemployment (Pearson correlation, $\Delta E^{*}$ and log-transformed $\left.E(0), r^{2}=-0.712, p=0.014, \alpha=0.05\right)$ and slower rates of increasing $\Delta E^{*}$ with increasing $R_{0}$ (Pearson correlation, $r^{2}=-0.736, p=0.01$ ). Neither logistic coefficient was significantly correlated with SES age structure.

We searched for SES structural features that might underlie the dependence of unemployment on system size using a principal components analysis (PCA) of initial sector employment, that is, the division of the employed labor force among sectors. This revealed an allometric contrast between the Agriculture sector, which tends to be a relatively larger sector in smaller SESs, and all other sectors (Fig. 3; Table S2). $\Delta E^{*}$ is correlated significantly with the allometric vector, PC 1 (Pearson correlation, $r^{2}=-0.774, p=0.009$; PC $1=79.09 \%$ of overall variance); growth rate of $\Delta E^{*}$ and $R_{0}$ are also significantly correlated (Pearson correlation, $r^{2}=-0.78, p=0.008$ ). PC 1 is itself significantly correlated with system size (Pearson correlation, $r^{2}=0.997, p<0.00001$ ), demonstrating that much of the size-correlated variation of relative unemployment, $\Delta E^{*}$, among SESs is the result of relative sector employment levels. Thus larger SESs are predicted to be more robust against disease-driven unemployment because of the general structural relationships among industries within the United States economy.

Those relationships also dictate the magnitude of the impact of outbreaks on a sector, and the sector's contribution to total SES unemployment. Relative sector unemployment, which varied greatly among SESs (Fig. S1), depends significantly on SES structure, demonstrated with a multivariate regression of relative unemployment per sector per SES on SES PC1 scores (all coefficients significant, $p<0.05$ ). Notably, several sectors show no significant relationship between unemployment and system structure, including Retail Trade, Education and Health Services, Leisure and Hospitality, and Other Services. Those sectors have among the weakest interactions ( $\mu$ in Eq. 3; see Materials and Methods), whereas for re- 
A

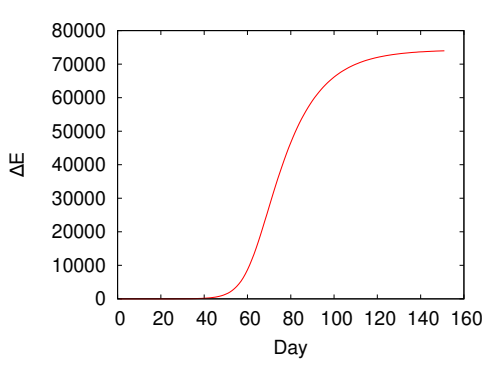

$\mathrm{D}$

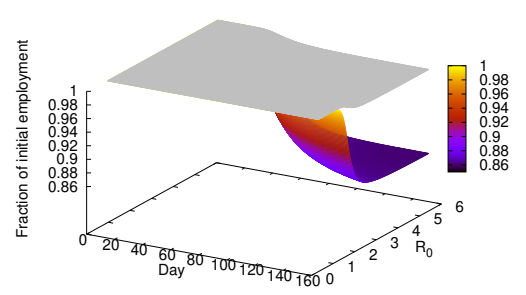

B

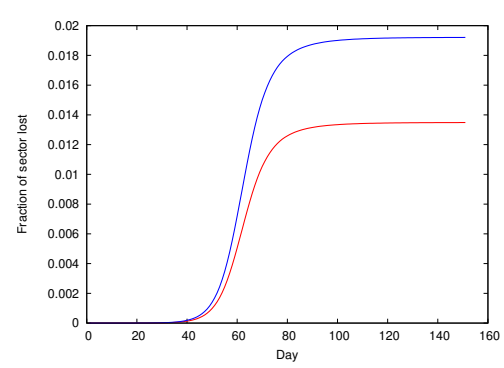

$\mathbf{E}$

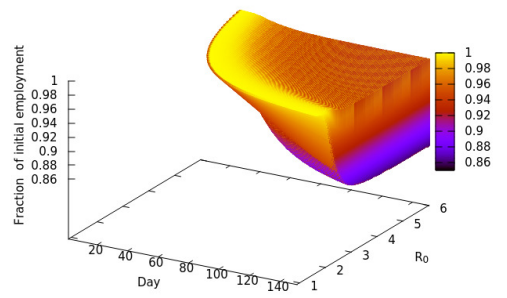

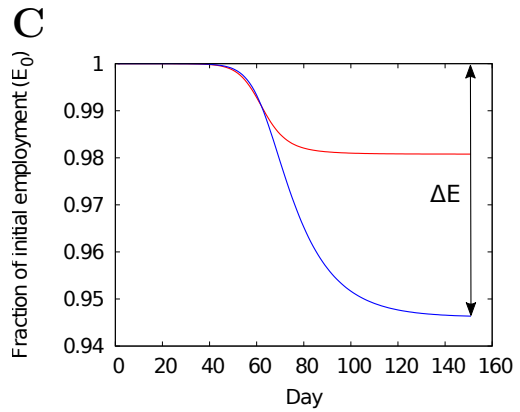

$\mathbf{F}$

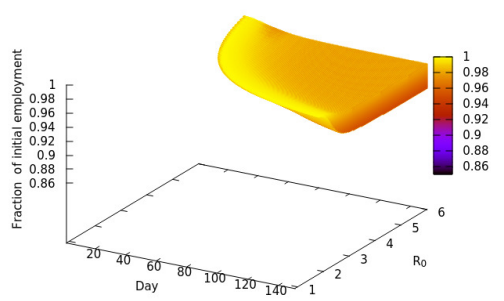

Figure 2: (A) Rising disease-caused (severe illness or mortality) loss of workers over time as infection sweeps through an employed labor force. Simulation is of the Los AngelesGlendale-Long Beach SES at a transmission rate $R_{0}=4.0 . \Delta E$ is the difference between initial employment (February, 2020) and employment at time $t$. Individual sectors differ according to age demographics and hence susceptibility to the disease $(B)$, for example Leisure and hospitality (red plot) and Manufacturing (blue plot). Manufacturing, which has a greater proportion of older workers, undergoes a more rapidly increasing and greater decline. Disease-driven losses (y-axis) are standardized by the initial employment of each sector. $(C)$ Economic cascade of unemployment (blue plot) driven by losses to the disease (red plot) for the Los Angeles-Glendale-Long Beach SES $(B)$. ( $D$ Total dynamics of all sectors for the Fresno SES over a $R_{0}$ range of 0.9-6.0. Upper grey surface is disease-caused losses, and lower surface shows the resulting economic cascades. Color spectrum is the fraction of initial total employment (February, 2020). (E) visualizes the magnitude of the cascade, and the gap between disease-caused and total reductions of employment, that is, the gap between the surfaces in $(D) .(F)$ is the equivalent visualization for the Los Angeles-Glendale-Long Beach SES, showing the different responses of this SES and Fresno. 
maining sectors, both the strengths of the dependencies and the ranking of sectors by those dependencies, are significantly correlated with the average interaction strength of a sector (Pearson's correlation, $r^{2}=-0.95, p=0.0001$; Spearman's rank correlation, $\rho=-0.878$, $p=0.0018)$. The most sensitive sector, Manufacturing, also interacts most strongly with other sectors (Fig. 1; Dataset S2). Thus, the response of a sector is dependent on both the structure of the specific SES, including its size and the apportionment of labor among sectors, as well as the relationships among sectors in the general economy of the United States.

The mid-point of the logistic relationship between $\Delta E^{*}$ and $R_{0}$ (Fig. S5) is the point at which a change of $R_{0}$ generates the largest change in the economic cascade. SES midpoints are significantly correlated with PC 1 (Pearson correlation, $r^{2}=0.777, p=0.008$ ) and range from 2.33-2.37, with larger SESs having higher thresholds of $R_{0}$ (Table $\mathrm{S} 1$ ) below which the cascading effects of outbreaks are relatively smaller. 2.3-2.4 is therefore an economically critical threshold of outbreak intensity for the California SESs. Actual California $R_{0}$ estimates for all the SESs (3) suggest that outbreaks in California would have sustained positive growth $\left(R_{0}>1\right.$ ), ranging between 1.59 (San Jose-Sunnyvale-Santa Clara) to 2.63 (Stockton-Lodi) at the beginning of March, 2020 (Table S3). The disease subsequently followed more complicated patterns because of measures intended to mitigate transmission, as well as variable societal adherence to those measures. Included in the measures were the mandated closures of non-essential workplaces, which contributed to record-breaking job losses. The business-as-usual model shows that reductions of employment, however, are an unavoidable consequence of the disease, even if workplaces remain open, and include workers lost to both the disease, and to the cascading secondary negative economic impacts of those primary casualties. The model also projects what the losses would be at a particular value of $R_{0}$ in the absence of mitigating measures. The actual $R_{0}$ values of the SESs imply that many of the systems would have encountered the tipping points observed at greater outbreak intensities in the model (Fig. 2D; Fig. S6). However, the real-world divergence of $R_{\text {eff }}$ from $R_{0}$ in all the SESs, a direct consequence of mitigation efforts, resulted in all systems avoiding the dramatic tipping points and cascades of declining employment predicted by the CASES model (Fig. 3B, C).

This raises the question of how well the economy might have fared if a business-as-usual approach had been adopted. We framed this question on the scale of long-term unemployment (6-12 months) by extending model duration to 365 days, and simulated at the actual SES $R_{0}$ values. Viewing all the SESs collectively by summing their model results, total unemployment would have reached 226,608 by August 1, 2020 (a 1.63\% unemployment rate relative to March 1, 2020), and by March, 2021 is forecast to be 756,152 or $5.43 \%$ of total SES employment at the onset of the pandemic (Fig. 4A). As of August, 2020, true total unemployment for the SESs was actually 1.5 million (10.77\%); a business-as-usual approach would therefore have spared $\approx 1.27$ million jobs. The forecast is also less than unemployment at the height of the Great Recession, when California's total unemployment rate surpassed $12 \%$ in 2010 (1). It would nevertheless be among the highest unemployment rates since the Great Recession. Overall, if COVID-19-driven unemployment in California has already peaked, and a potential recovery began during the summer of 2020 (23), then the total unemployment generated by a business-as-usual approach would be less than unemployment already incurred under mitigation conditions (Fig. 4A). A similar conclusion was reached 
A
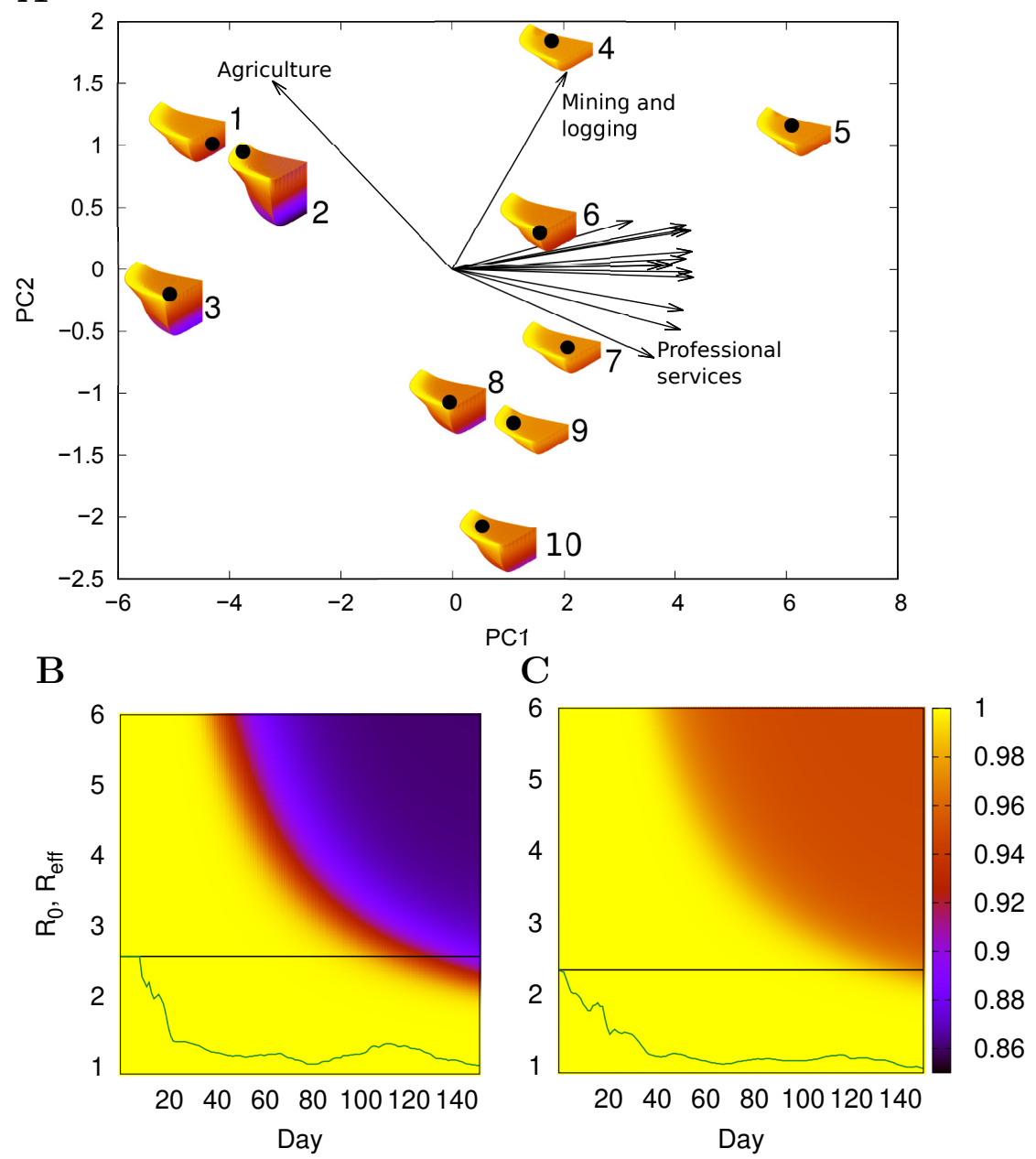

Figure 3: (A) Principal comopnents analysis ordination of SESs by employment levels per sector, February, 2020. Each SES is represented by a visualization of its economic cascade under the business-as-usual model, equivalent to examples illustrated in Figs. $2 \mathrm{E}$ and F. Arrows show the contribution of eaxh sector ( $\mathrm{PC}$ loadings) as vectors in the ordination space. PC 1 is thus show to be a contrast between the Agriculture sector and all others, while PC 2 is a gradient of increasing goods producing versus services providing sectors. Arrow length is proportional to the influence of the sector on the ordination. SESs: 1 - Oxnard-Thousand Oaks-Ventura; 2 - Fresno; 3 - Stockton-Lodi; 4 - Riverside-San Bernadino-Ontario; 5 - Los Angeles-Glendale-Long Beach; 6 - San Diego-Carlsbad; 7 - Anaheim-Santa Ana-Irvine; 8 San Jose-Sunnyvale-Santa Clara; 9 - Oakland-Berkeley-Livermore; 10 - San Francisco-San Mateo-Redwood City. $B$ and $C-R_{0}$ and $R_{\text {eff }}$ trajectories superimposed on business-as-usual model forecasts of SES relative unemployment (color spectrum). $R_{0}$ trajectories (straight black line) indicate model outcome if transmission rates on March 1, 2020 had remained unchanged, whereas $R_{\text {eff }}$ trajectories (green lines) show actual SES histories. $(B)$ Fresno, $(C)$ Los Angeles-Glendale-Long Beach. 
by earlier work on the predicted economic impact of an influenza pandemic on the United Kingdom's economy (13). The total cost of a business-as-usual scenario, however, is greater than indicated by this simplistic comparison of model and real unemployment. There are two additional cost dimensions to be considered.

First, unemployment would be distributed disproportionately among the SESs relative to the sizes of their employed populations. Real unemployment exceeded model forecasts in most SESs (Fig. S7), consistent with the collective result explained above. For example, by August, 2020 true unemployment in the San Francisco-San Mateo-Redwood City SES exceeded the model estimate by 43,433 (Fig. 4B). Smaller SESs however, which in many instances correspond to California's inland cities, would in general respond differently. Both Fresno (Fig. 4C) and Stockton-Lodi (Fig. S6), systems forecast by the PCA to be most vulnerable to disease-driven cascading unemployment (Fig. 3), had unemployment levels by August, 2020 less than simulated unemployment. This suggests that for those SESs, a business-as-usual scenario would have been worse than the real-world experience. OxnardThousand Oaks-Ventura, the other system predicted by the PCA to be vulnerable to cascades (Fig. 3), experienced August unemployment matched almost exactly by the model prediction; 305,700 and 306,747 respectively (Fig. 4D). It appears that either strategy would produce a similar result for that particular SES, but in general, SES responses are variable enough to support mitigation policies that pay attention to the variability among SESs based on size and economic structure. Unemployment generated by a business-as-usual approach would have mixed impact throughout the state, benefiting some regions, while costing others. This is consistent with arguments at the state level that it is important to consider geographically correlated variation when examining the economic impacts of COVID-19 (22).

Second, an unmitigated business-as-usual approach to the disease, while sparing jobs, would incur significant losses of life and health. Ultimately, the scenario's economic downturn is driven by workers who die or become too ill to continue or return to work, with simulated totals of 16,825 by August 1, 2020, and 169,967 by March, 2021, including 16,774 and 169,450 fatalities respectively. Scenario unemployment is dominated by death because of the disparities of case fatality rates among age categories: a majority of younger workers who contract the disease recover fully, whereas a majority of older workers experience severe illness or death. The totals are also based on workers only, and do not account for illness and mortality in the broader SES populations. As of October, 2020, actual total COVID-19 related deaths in California stood at $\approx 17.5$ thousand. By comparison, COVID-19 related deaths in New York City exceeded 19,000 over the same period of time. In contrast to the un-quantifiable ethical and sociological costs of an unabated pandemic, the economic cost of a business-as-usual policy can be estimated using Value of Statistical Life (VSL) estimates (United States Office of Management and Budget 2003). VSL estimates allow us to speculate what the scenario should cost if employees worked in an increasingly risky environment (29). Current VSL estimates align roughly at $\$ 10$ million per person (8). The estimated cost to the California economy under a business-as-usual scenario would therefore have been $\$ 167.7$ million by August, 2020, increasing dramatically to $\$ 16.94$ billion by March, 2021 .

There are assumptions and nuances of the model that constrain its applicability. Data uncertainty and unavailability mean that model forecasts should not be treated as precise numerical predictions, although we assert the qualitative accuracy of our conclusions. Further- 
A

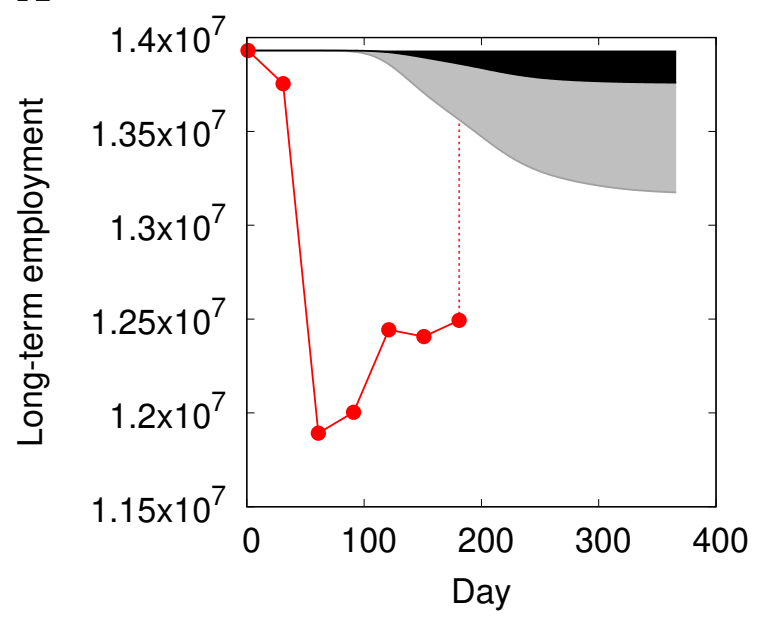

C

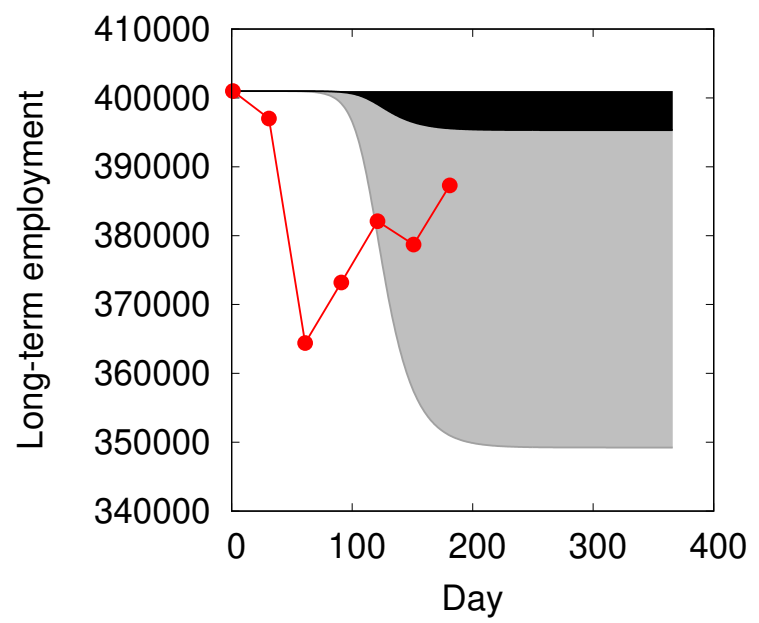

B

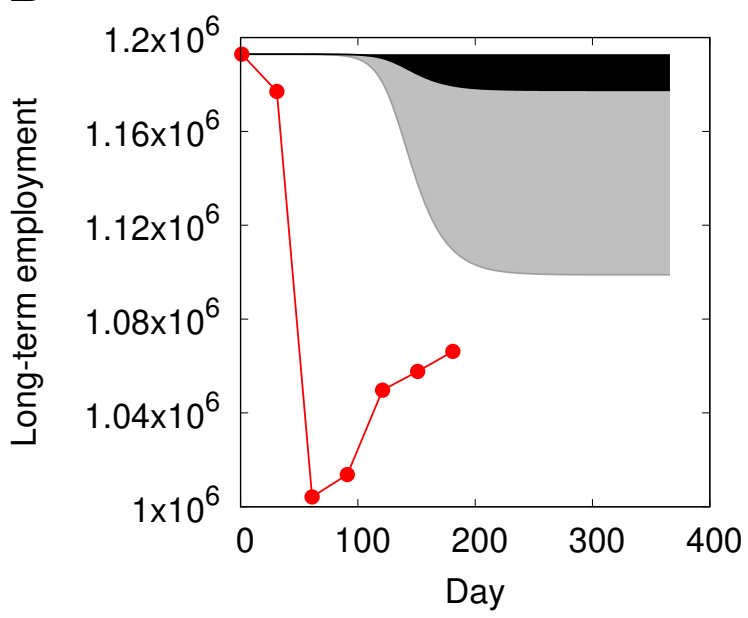

$\mathrm{D}$

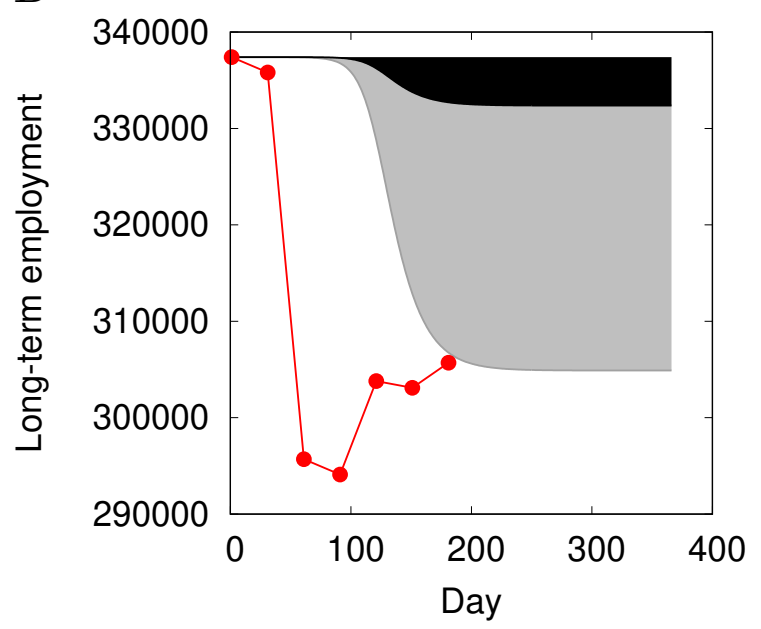

Figure 4: Comparisons of unemployment forecast by the business-as-usual model and actual unemployment of March to August, 2020. (A) - all SESs combined; $(B)$ - San Francisco-San Mateo-Redwood City; $(C)$ - Fresno; $(D)$ - Oxnard-Thousand Oaks-Ventura. Actual unemployment is depicted by solid red lines, connecting actual monthly estimates (red circles). Model forecast unemployment is the solid grey line. Model unemployment is partitioned between losses to the disease because of death or long-term illness, and unemployment caused by the cascading economic consequences; black and grey filled areas respectively. Dotted red lines illustrate the gap between actual unemployment and model forecasts respectively. 
more, SESs are treated as independent entities, when in fact they are parts of an integrated and nested economy at the state, national and global levels. Nevertheless, our analysis shows that there are important structural differences and economic responses among the SESs that would be missed if SESs were aggregated at higher organizational or geographic levels. SES variation in fact suggests that combining local SES structural data with dynamic epidemiological and economic models could form the basis for flexible policies that are strategically designed to disrupt the spread of pandemics, and simultaneously adhere to the highest and most compassionate moral and societal standards, while minimizing inevitable economic damage. California has adopted a county by county shutdown strategy based on positive case counts, a strategy suggested to be epidemiologically effective (12). Our work supports the incorporation of similar strategies to manage economic impacts.

Simplistic prioritization of the economy over the health emergency, while possibly lessening economic losses, would nevertheless result in a significant recession, coupled with greatly increased health consequences of the disease. Fewer jobs would have been lost in California had business continued as usual, but at the expense of the health and lives of tens of thousands of workers. In addition to the moral and societal costs of that impact, future recovery would be hampered by a smaller, younger and less experienced post-pandemic labor force.

\section{Materials and Methods}

\section{SES networks}

Each SES was represented as a network of industrial sectors linked by the exchange of goods and services between sectors (Fig. 1). The sectors represent the major goods producing and services providing sectors within the North America Industry Classification System as utilized by the USBLS and California Employment and Development Department (Dataset S2). These particular socio-economic systems were selected primarily because they have reported monthly employment data for the major fifteen industrial sectors used by the USBLS to aggregate economic activity in the United States during the relevant time interval (February-August, 2020). We used seasonally unadjusted data (27, 28) (Table S1), and included the Agriculture industrial sector (Total farm) both for completeness of the data, and the importance of, albeit relatively smaller, farming components of the SESs.

The SES network model represents each industrial sector as a node (for example, 18, 32). Nodes are linked by exchanges of goods and services, and the network is a complete graph because all sectors interact economically. The inter-sector links are bi-directional, representing the generally asymmetric requirements between sectors. Link weights, which represent the strengths of the interactions between two sectors, are derived from the value of inter-industry exchanges for the United States during 2019 (US Bureau of Economic Analysis; Dataset S3), and based ultimately on input-output evaluations (16). The CASES model is based on a standardized total sector requirement, and therefore all the requirements of a sector, including intra-sector trade, sum to 1.0. Link weights are thus calculated as

$$
w_{i j}=\frac{q_{i j}}{\sum_{n=1}^{S}\left(q_{i n}+q_{n i}\right)}
$$


where $q_{i j}$ is the inter-industry exchange value of the requirements of sector $i$ of sector $j, q_{j i}$ represents the requirements of $j$ of $i$, and $S$ is the number of sectors. The sector by sector asymmetric matrix $I$ comprises the elements $w_{i j}$ and $w_{j i}$.

\section{Model derivation}

The CASES model proposes that employment in sector $i$ responds when external drivers remove or add workers, or labor, to the sector. In the case of the business-as-usual scenario for the pandemic we consider negative drivers only, that is, drivers that remove workers, specifically mortality and severe illness. If we represent those drivers collectively as the rate at which a fraction of the workforce is removed, $\phi_{i}$, then the rate of change of employment is

$$
\frac{d E_{i}}{d t}=-\phi_{i} E_{i}
$$

where $E_{i}$ is employment in sector $i . E_{i}$, however, is also a function of employment in other sectors, and disease-driven reductions of $E$ in any sector therefore leads to further unemployment as productivity declines.

The model thus accounts for the mutual dependencies within the inter-sector network, with those dependencies being partitioned into three types: intra-sector, requirements of sector $i$ of other sectors, and requirements of other sectors of sector $i$. Intra-sector requirements are the diagonal elements of $I, w_{i i}$, and inter-sector requirements are rows and columns of $I$, the elements of which are denoted by $w_{i j}$ and $w_{j i}$ respectively. A complete model which captures the network relationships of a node or sector is

$$
\frac{d E_{i}}{d t}=-\phi_{i} E_{i}\left[w_{i i}+\sum_{j=1}^{S}\left(w_{i j} \frac{E_{j}}{E_{j}(0)}+w_{j i} \frac{E_{j}}{E_{j}(o)}\right)\right]
$$

where $S$ is the number of sectors, and the terms within parentheses are inter-node exchanges. The contributions of other sectors to $E_{i}$ are relative to their own initial employment levels $\left(E_{j} / E_{j}(0)\right)$, and are weighted by the sector interactions $(w)$. Equation 3 is simplified to

$$
\frac{d E_{i}}{d t}=-\phi_{i} E_{i}\left[w_{i i}+\left(\sum_{j=1}^{S} \frac{\mu_{i j} E_{j}}{E_{j}(0)}\right)\right]
$$

where $\mu_{i j}$ is the sum of $w_{i j}$ and $w_{j i}$. Recall that $\phi_{i} \leq 0$, and hence $d E_{i} / d t \leq 0$, a simplification that reflects the business-as-usual model and ignores any recovery by exclusion of terms for hirings, which is assumed to remain constant throughout the scenario. Sectors and the system thus remain in equilibrium if there is no disease, because $\phi=0$ for all $i$.

\section{Coupled SIR-CASES model}

Mortality rates and rates of severe illness are fractions of $E_{i}(t)$, and are combined additively to yield $\phi_{i}$. Those fractions themselves are obtained using a SIR (Susceptible, Infected, Removed) epidemiological model (14). The SIR model is the most basic compartmental 
epidemiological model providing time series estimates of the number of people in a population who have completed the infectious phase either because of survival or death. Survival may refer to a range of conditions, including long-term disablement. The SIR model consists of three coupled ordinary differential equations,

$$
\begin{aligned}
\frac{d S}{d t} & =-\frac{\beta I S}{N} \\
\frac{d I}{d t} & =\frac{\beta I S}{N}-\gamma I \\
\frac{d R}{d t} & =\gamma I
\end{aligned}
$$

where $S$ is the number of susceptible individuals in the population, $I$ is the number infected, and $R$ the number removed because of death or recovery (not to be confused with the unfortunate duplicate use of $R$ to signify growth rate of the epidemic, that is, $R_{0}$ and $R_{\text {eff }}$ ); $N$ is the total population size. $\beta$ is the average number of contacts per unit time, and here is estimated as the quotient of $R_{0}$ and the average duration of the disease, set here at 14 days; $\beta=R_{0} / 14 . \gamma$ is the rate at which infectious individuals are removed, estimated here as the inverse of the average time for which infected individuals are assumed to be infectious, in this case 10 days (US Centers for Disease Control and Prevention).

Each SES examined in the study has an estimated $R_{0}$ for March 1, 2020, based on ensemble estimates from the California Department of Public Health's California COVID Assessment Tool (CalCAT) (26), derived from COVID Act Now (10), COVID19 Projections.com (9), the University of California Los Angeles (33), LEMMA (24), Johns Hopkins University (15), Stanford University (7), University of California San Francisco (31), and Harvard University (17) models (Table S5). Ensemble estimates were either available for each SES, or were taken from estimates for the demographically largest county in an SES (Table S3).

The growth rate of the disease is not constant during a typical epidemic, and can respond to numerous factors, such as changes of virulence, and societal measures, including improving treatments, social distancing and quarantines. Here we assumed $R_{0}$ to be constant during a simulation because the business-as-usual scenario asserts that no mitigation measures are taken to reduce it. We assume a constant contagiousness of the SARS-CoV-2 virus, although there is recent evidence that a more infectious strain might have swept through populations since the start of the pandemic $(21,11,30)$.

\section{Model simulation}

The parameterization of a SIR model for each SES, given a specific value of $R_{0}$ allows estimation, from the time series of the Removed component, of the number of individuals who have died, or were hospitalized because of severed illness, using nationwide case fatality rates and rates of hospitalization for severe infections (Table S4). The Removed component in the SIR-CASES model is derived from the fraction of an SES's population that was employed in February, 2020, and it is assumed that COVID-19 dynamics among employed persons is the same as those for the general population. The component is further decomposed according to the mortality and hospitalization rates of four age categories (Table S4). 
Age classes used for tracking by the United States Centers for Disease Control are not commensurate with those used by the USBLS for worker ages, so the USCB data were aggregated in the following manner to obtain rates for the coupled SIR-CASES model. The model compartment for ages 5-17 years used USCB data for workers 17 years and younger; 18-49 years aggregated data from USCB category 18-44 years plus half the number of workers recorded for the 45-54 years category; the 50-64 years compartment aggregated data of half the number recorded by the USCB for the 45-54 years category plus the 55-64 years category; and the 65 years plus compartment was equal to the 65 years and over USCB category. Data used were specific to each SES, and were used to generate a matrix of age fractions per compartment per industrial sector for each SES.

A complete model flow therefore begins with the calculation of how many workers are lost from each age compartment of a sector by decomposing the SIR Removed compartment according to sector demographics. The CASES dynamics of each sector's age compartment are then calculated according to Eq. 4, and a sector's total dynamics may be written in a properly expanded form as

$$
\frac{d E_{i}}{d t}=-\sum_{n=1}^{4}\left[E_{i, n} \omega_{i, n}\left[w_{i i}+\sum_{j=1}^{15}\left(\frac{\mu_{i j} E_{j}}{E_{j}(0)}\right)\right]\right]
$$

where $n$ is an age compartment.

We consider March 1, 2020 to be very early in the outbreaks, and set an initial fraction of the population that was infected in each SES at 0.00002. Thus, at the beginning of a scenario the Susceptible fraction of the population is 0.99998, the Infected fraction is 0.00002 , and the Removed fraction is zero. An outbreak at any time $t>0$ implies then that $\phi_{i}>0$, and all sectors are affected by both a direct impact on $E_{i}$, and a cascading impact through the SES network. The cascade is a function of weighted inter-sectoral interactions, sector employment levels, and the age structures of sector populations. A cascade captures the expected changes to employment within a sector as trade both within and between sectors decline because of lowered productivity, supplies and demands, driven by the loss of workers.

ACKNOWLEDGMENTS.This study is supported by United States National Science Foundation grant 2032769 to PDR.

Supplementary Information. All supplemental information referred to in the text is available at https://github.com/calacademy-research/Roopnarine-et-al

\section{References}

[1] CAEDD, California unemployment drops to an even 11 percent in September, https://tinyurl.com/yaxhkwab, October 2020.

[2] L L L L Market Information for Metropolitan Areas, https://tinyurl.com/yaeync44, 2020.

[3] CalCAT, Current R-effective in California, https://calcat.covid19.ca.gov/cacovidmodels/, 2020. 
[4] Kieran Corcoran, California's economy is now the 5th-biggest in the world, and has overtaken the United Kingdom, Business Insider (2018), https://www.businessinsider.com/california-economy-ranks-5th-in-the-world-beatingthe-uk-2018-5.

[5] International Monetary Fund, World Economic Outlook, October 2020: A Long and Difficult Ascent, https://www.imf.org/en/Publications/WEO/Issues/2020/09/30/worldeconomic-outlook-october-2020, October 2020.

[6] Thomas A Garrett, Pandemic economics: The 1918 influenza and its modern-day implications, Federal Reserve Bank of St. Louis Review 90 (2008), no. March/April 2008.

[7] Jeremy Goldhaber-Fiebert, Fernando Alarid-Escudero, and Jason Andrews, StanfordCIDE Coronavirus Simulation Model (SC-COSMO) -Technical Description Document, Version 1.0, https://tinyurl.com/ya4rdhys, 2020.

[8] Michael Greenstone and Vishan Nigam, Does social distancing matter?, University of Chicago, Becker Friedman Institute for Economics Working Paper (2020), no. 2020-26.

[9] Youyang Gu, Model details, https://tinyurl.com/y83gm22f, 2020.

[10] Max Henderson, Eric Carlson, Igor Kofman, Jonathan Kreiss-Tomkins, and Anna Blech, Covid Act Now Assumptions And References, https://tinyurl.com/yb8kadkz, 2020.

[11] Yixuan J Hou, Shiho Chiba, Peter Halfmann, Camille Ehre, Makoto Kuroda, Kenneth H Dinnon, Sarah R Leist, Alexandra Schäfer, Noriko Nakajima, Kenta Takahashi, et al., SARS-CoV-2 D614G variant exhibits efficient replication ex vivo and transmission in vivo, Science 370 (2020), no. 6523, 1464-1468.

[12] Vadim A Karatayev, Madhur Anand, and Chris T Bauch, Local lockdowns outperform global lockdown on the far side of the COVID-19 epidemic curve, Proceedings of the National Academy of Sciences 117 (2020), no. 39, 24575-24580.

[13] Marcus R Keogh-Brown, Simon Wren-Lewis, W John Edmunds, Philippe Beutels, and Richard D Smith, The possible macroeconomic impact on the UK of an influenza pandemic, Health economics 19 (2010), no. 11, 1345-1360.

[14] William Ogilvy Kermack and Anderson G McKendrick, A contribution to the mathematical theory of epidemics, Proceedings of the Royal Society of London. Series A 115 (1927), no. 772, 700-721.

[15] Joseph Chadi Lemaitre, Kyra H Grantz, Joshua Kaminsky, Hannah R Meredith, Shaun A Truelove, Stephen A Lauer, Lindsay T Keegan, Sam Shah, Josh Wills, Kathryn Kaminsky, Javier Perez-Saez, Justin Lessler, and Elizabeth C Lee, A scenario modeling pipeline for COVID-19 emergency planning, medRxiv (2020).

[16] WW Leontief, S Fabricant, I Friend, T Koopmans, O Morgenstern, W Jacobs, M Hoffenberg, and R Goldsmith, Input-output analysis and its use in peace and war economics, American Economic Review 5 (1949), 211-225. 
[17] Xihong Lin, Visualizing COVID-19's Effective Reproduction Number (Rt), http://metrics.covid19-analysis.org/, 2020.

[18] James McNerney, Brian D Fath, and Gerald Silverberg, Network structure of interindustry flows, Physica A: Statistical Mechanics and its Applications 392 (2013), no. 24, 6427-6441.

[19] Maria Nicola, Zaid Alsafi, Catrin Sohrabi, Ahmed Kerwan, Ahmed Al-Jabir, Christos Iosifidis, Maliha Agha, and Riaz Agha, The socio-economic implications of the coronavirus pandemic (COVID-19): A review, International journal of surgery (London, England) 78 (2020), 185.

[20] OMB, OMB BULLETIN NO. 20-01, https://tinyurl.com/y7oz7sfy, March 2020.

[21] Jessica A Plante, Yang Liu, Jianying Liu, Hongjie Xia, Bryan A Johnson, Kumari G Lokugamage, Xianwen Zhang, Antonio E Muruato, Jing Zou, Camila R Fontes-Garfias, et al., Spike mutation D614G alters SARS-CoV-2 fitness, Nature (2020), 1-9.

[22] Maria Polyakova, Geoffrey Kocks, Victoria Udalova, and Amy Finkelstein, Initial economic damage from the COVID-19 pandemic in the United States is more widespread across ages and geographies than initial mortality impacts, Proceedings of the National Academy of Sciences 117 (2020), no. 45, 27934-27939.

[23] Mark Schniepp, Status of the Recovery / Summer 2020, https://californiaforecast.com/july-2020/, July 2020.

[24] Joshua Schwab, LEMMA (Local Epidemic Modeling for Management Action), https://tinyurl.com/yag8o9wy, 2020.

[25] Adam Sheridan, Asger Lau Andersen, Emil Toft Hansen, and Niels Johannesen, Social distancing laws cause only small losses of economic activity during the COVID-19 pandemic in Scandinavia, Proceedings of the National Academy of Sciences 117 (2020), no. 34, 20468-20473.

[26] California COVID Assessment Tool, Modeling COVID-19 to Inform State and Local Response, https://tinyurl.com/y2yzakgy, 2020.

[27] USBLS, Monthly Employment Situation Report: Quick Guide to Methods and Measurement Issues, https://www.bls.gov/bls/empsitquickguide.htm, 2020.

[28] - Technical Notes for the Current Employment Statistics Survey, https://tinyurl.com/y7bzsy7s, 2020.

[29] W Kip Viscusi and Joseph E Aldy, The value of a statistical life: a critical review of market estimates throughout the world, Journal of risk and uncertainty 27 (2003), no. 1, $5-76$. 
[30] Erik Volz, Verity Hill, John T McCrone, Anna Price, David Jorgensen, Áine O'Toole, Joel Southgate, Robert Johnson, Ben Jackson, Fabricia F Nascimento, et al., Evaluating the effects of SARS-CoV-2 Spike mutation D614G on transmissibility and pathogenicity, Cell (2020).

[31] Lee Worden, Rae Wannier, Micaela Neus, Jennifer C. Kwan, Alex Y. Ge, Eugene T. Richardson, and Travis Porco, Estimation of COVID-19 transmission rates in California and the U.S. with reporting delays, medRxiv (2020).

[32] Ming Xu and Sai Liang, Input-output networks offer new insights of economic structure, Physica A: Statistical Mechanics and its Applications 527 (2019), 121178.

[33] Difan Zou, Lingxiao Wang, Pan Xu, Jinghui Chen, Weitong Zhang, and Quanquan Gu, Epidemic model guided machine learning for COVID-19 forecasts in the United States, medRxiv (2020). 